\title{
e-Phaïstos
}

e-Phaïstos

Revue d'histoire des techniques / Journal of the history

of technology

IV-2 | 2015

Patrimoines de l'eau

\section{Représentations et usages des armements contemporains : pour une socio-anthropologie de la complexité technique}

Sophie Lefeez

\section{(2) OpenEdition}

1 Journals

Édition électronique

URL : http://journals.openedition.org/ephaistos/813

DOI : 10.4000/ephaistos.813

ISSN : 2552-0741

Éditeur

IHMC - Institut d'histoire moderne et contemporaine (UMR 8066)

Édition imprimée

Date de publication : 1 octobre 2015

Pagination : 105-110

ISSN : 2262-7340

\section{Référence électronique}

Sophie Lefeez, «Représentations et usages des armements contemporains : pour une socioanthropologie de la complexité technique », e-Phaïstos [En ligne], IV-2 | 2015, mis en ligne le 30 novembre 2016, consulté le 15 septembre 2020. URL : http://journals.openedition.org/ephaistos/813 


\title{
Représentations et usages des armements contemporains : pour une socio-anthropologie de la complexité technique.
}

\author{
Thèse de doctorat de philosophie \\ Université Paris 1 Panthéon-Sorbonne en partenariat avec le Centre d'étude des techniques, des \\ connaissances et des pratiques (CETCOPRA) \\ Soutenue le 12 septembre 2014 \\ Un volume (557 p.)
}

Directeur de thèse :

Gérard DUBEY, professeur à l'Institut MinesTélécom

Jury:

Charles KIRKE, senior lecturer military anthropology and human factors, department of management and security, Cranfield University (Royaume-Uni)

Jean-Luc METZGER, chercheur à Orange Labs, associé au CNAM-LISE et au centre Pierre Naville (rapporteur)

Beatriz RIOFRIO-GONZALEZ, département de la stratégie d'Airbus, manager "regional and military derivatives", anciennement manager "product strategy military"

Victor SCARDIGLI, directeur de recherche émérite au CNRS (président du jury)

Pascal VENNESSON, professeur à la Nanyang Technological University, détaché de l'université Panthéon-Assas Paris II (rapporteur)
Monsieur le Président,

Madame et Messieurs les membres du jury,

Je suis très honorée de pouvoir présenter devant vous cette thèse de doctorat portant sur les choix techniques dans les matériels militaires. Les origines de ce travail prennent leurs racines presque deux ans avant ma première inscription en doctorat. J'étais alors responsable du comité Armée du Futur au sein de l'ANAJ-IHEDN, l'association des auditeurs jeunes de l'Institut des hautes études de défense nationale. Dans le cadre de notre travail sur la contre-insurrection, je me suis étonnée de la capacité des insurgés à mettre en défaut, à l'aide de moyens simples, des armements à la pointe de la technique. Ces images de véhicules blindés sautant sur des explosifs artisanaux suscitaient en moi deux interrogations persistantes: quelle guerre les armées et les ingénieurs avaient-ils en tête lorsqu'ils ont conçu ces matériels pour ne pas avoir anticipé un tel type de menace ? Et pourquoi cette obsession pour la haute technicité ${ }^{1}$ dans notre société quand des matériels de plus faible technicité peuvent se montrer efficace - du moins parfois ?

Deux autres observations renforçaient mon intérêt: d'une part, vouloir un équipement à la pointe de la technique requiert un certain temps de 
$\mathrm{R} \& \mathrm{D}$, ce qui contribue à une durée de programmes d'armement comprise entre quinze et vingt ans ; sachant que la durée de vie d'un matériel peut aller jusqu'à quarante ans, cela veut dire qu'il faut penser les matériels jusqu'à soixante ans en avance - une gageure. D'autre part, l'avantage technique a également un prix. Le coût d'achat et de soutien des matériels militaires ne cesse de croître, à un rythme supérieur à celui du PIB : une impasse budgétaire se profile donc, et fait craindre, si l'on continue à opter pour des équipements de haute technicité, d'aboutir une armée « échantillonnaire ».

Comme cet attrait pour la haute technicité semblait étroitement liée aux représentations imaginaires du combat et de la technique, et qu'il existe peu d'études sur le sujet de l'armement en France, en-dehors du champ de l'ingénierie et de l'histoire, le CETCOPRA (Centre d'étude des techniques, des connaissances et des pratiques), pour qui la prise en compte de l'imaginaire des techniques et un travail de terrain sont des caractéristiques identitaires fortes, était un laboratoire d'accueil approprié pour faire mûrir cette recherche, d'autant que les matériels militaires y avaient déjà fait l'objet d'études. De plus, ce laboratoire a pour tradition d'inclure dans ses recherches les différents corps de métier gravitant autour d'un objet technique, comme ce fut le cas pour l'avion (avion commercial et avion de chasse), et de tenir compte de l'aspect soutien, souvent négligé dans les études sur les objets techniques si l'on en croit David Edgerton². J'ai souhaité avoir la même approche intégrée concernant les matériels de guerre.

En effet, la plupart des études sur l'armement se rangent, comme celle de Donald McKenzie 3 ou William Genieys 4 , dans la sociologie des organisations ou la sociologie politique, et entendent, dans une démarche compréhensive et explicative, décrire le processus d'interactions entre les instances décisionnelles privées et publiques ayant conduit à la mise au point du matériel étudié. En outre, les travaux sur l'armement français portent généralement soit sur la conception (tels l'ouvrage de Genyies et les documents du Centre des hautes études de l'armement), soit sur l'usage (comme les travaux du CETCOPRA). Face à cela, mon approche se veut très " micro ", et ambitionne d'associer, dans un même mouvement, usage et conception de manière à appréhender l'ensemble $\mathrm{du}$ processus technique 5 , ainsi que de mettre au jour l'imaginaire des usages à travers une comparaison entre les usages anticipés lors de la conception et les usages réels.

À cet effet, j'ai choisi de prendre en étude de cas un matériel en fin de vie: le système de missile antichar Milan pour fantassin débarqué. Au cours de mon enquête, il fut remplacé par un matériel plus moderne: le système de missile antichar Javelin, de fabrication américaine. Cela me donna ainsi l'occasion de réaliser une comparaison au plan de l'appropriation, de l'usage et plus particulièrement de la capacité de réinvention, entre un matériel ancien et un autre plus moderne, doté d'automatismes et de techniques numériques. Cette étude de cas se veut être un approfondissement et non le cœur du sujet de l'usage, car mon souhait était d'analyser les matériels en général. C'est pourquoi cette étude fut conduite en ayant constamment en tête l'ensemble des matériels. Ce fut ma façon de lier le particulier au général, et le général au particulier.

Mon intention était de comprendre de «l'intérieur», à partir des représentations des acteurs, ce qui se déroule dans la phase de conception et comment les armements sont utilisés, afin de le rendre compréhensible aux personnes non familières de ce milieu. Je me suis attachée à ne pas porter de jugement normatif ou éthique car, comme l'écrivit Max Weber 6 , le jugement moral ne dispense ni de la compréhension, ni de l'explication.

Pour accéder aux représentations et aux systèmes de croyances des enquêtés afin de dévoiler les motivations en faveur des matériels militaires high-tech, je me suis inscrite dans la socioanthropologie de Marcel Mauss7. Ma démarche fut 
donc qualitative et inductive, et mon analyse porta aussi bien sur les discours que sur les pratiques. La méthode consiste à partir sans grille d'analyse préalable, ce qui peut conduire à un foisonnement de résultats. Devant cette richesse, j'ai fait le choix d'en rendre compte plutôt que de me focaliser sur un axe majeur afin de donner à voir ce qui se joue dans l'acquisition des matériels, un sujet sur lequel il y a à ce jour peu de choses publiées en France.

Parallèlement à la sociologie de la conception, la sociologie des usages fut mobilisée pour étudier l'appropriation et la réinvention des matériels. Néanmoins, il s'agit moins d'étudier la conception elle-même, ou le processus d'appropriation, ou encore la réinvention des objets, que de comprendre comment ces éléments - et d'autres sont liés, car nous croyons avec Edgar Morin qu'il y a de la connaissance dans les inter-relations ${ }^{8}$. Et c'est précisément pour comprendre ce qui s'y joue que j'ai adopté une approche multidimensionnelle. Certains chapitres sont donc particulièrement socio-anthropologiques, tandis que d'autres entremêlent l'histoire et l'économie, ou s'inscrivent résolument dans la sociologie des techniques. Toutefois, la multi-dimensionnalité invite à tirer encore plus loin certains fils de la pelote, au risque de se perdre dans un dédale de chemins tous plus alléchants les uns que les autres pour une personne curieuse. Il me fallut ainsi renoncer à poursuivre certaines pistes, comme par exemple la multiplication des contraintes normatives pesant sur la conception.

En pratique, j'ai rencontré quatre groupes d'enquêtés: les trois groupes impliqués dans la conception des armements, autrement dit les services d'État-major, la direction générale de l'armement (DGA) et l'industrie de défense, puis les combattants, qui constituent le corps des usagers. $\mathrm{J}$ 'ai réalisé environ une centaine d'entretiens; les usagers comptent pour les plus nombreux vu la diversité des matériels que je souhaitais couvrir.

Il me faut ici signaler qu'il me fut difficile d'obtenir des entretiens de la part de la DGA. Cette réticence me semble traduire un profond mal-être, dont l'une des explications se situe dans le décalage entre les réformes subies depuis une vingtaine d'années et le sens que le personnel donne à son travail. En outre, l'institution faisant régulièrement l'objet de critiques de la part des militaires et des industriels, j'y vois également la crainte que mon enquête ne les expose à davantage de critiques. Pourtant, on lui reconnaît une véritable expertise technique. Cet apparent paradoxe illustre, à mon sens, sa difficulté de positionnement symbolique, et invite à réétudier sa place dans le processus d'acquisition.

Dans l'ensemble, ces enquêtes m'ont confrontée à deux cultures de métier: l'ingénierie et le militaire. Ma rencontre avec l'ingénierie engendra un choc épistémique car la méthode socioanthopologique se trouve aux antipodes des pratiques de ce milieu : elle n'a pas d'hypothèse $a$ priori, la démarche est inductive et non déductive, il est impossible de se tenir à une liste de tâches ou un calendrier prédéfinis car c'est le terrain qui les impose au chercheur, etc. Je crois néanmoins qu'une collaboration entre ingénieurs et chercheurs en sciences humaines et sociales, par-delà les différences méthodologiques, est nécessaire sur les questions d'armement pour l'enrichissement réciproque qu'elle permet. Quant au militaire, il constitua pour moi un embarquement affectif, me renvoyant à mon premier choix de carrière: les armes.

Ce sujet de recherche se révéla en effet personnel à plus d'un titre. Mes lectures en histoire et en philosophie des sciences m'ont également replongée dans mes questionnements estudiantins sur les présupposés épistémiques des sciences dures et de l'économie, sur la mathématisation du monde, ainsi que sur la recherche d'une objectivation comme condition de la connaissance, qui procède en réalité d'une dissociation d'avec le monde réel qu'on prétend chercher à connaître. Il me fallut donc procéder à une objectivation de mon vécu socioaffectif et historique afin de montrer aux lecteurs la 
subjectivité que j'ai mobilisée et expliciter les déterminations sociales qui se sont exercées sur ma pratique.

Une fois le cadre méthodologique fixé et les biais éventuels reconnus, je pouvais me lancer dans le recueil et l'analyse des données. Mes recherches ont montré que la quête de performance repose sur un processus social et non uniquement technicoopérationnel. Dans ce schéma mental où la guerre est perçue comme un affrontement de capacités, besoin, capacité et performance finissent par coïncider. Â travers l'analyse des représentations en phase de conception, la thèse parvint à la conclusion que la polyvalence, l'architecture systémique et la gestion sociale de l'incertitude étaient dès le départ liés dans l'esprit des concepteurs.

En approfondissant ces premiers résultats, la thèse mit en évidence la prégnance de la rationalité gestionnaire et technicienne - qui partagent des racines identiques. Toutes les deux érigent la productivité en idéal suprême, comme le relevait Hannah Arendt 9 . Dans sa dimension technique, cette rationalité attend de la recherche scientifique qu'elle accroisse la puissance: c'est la quête du " plus vite, plus haut, plus fort ». Dans le domaine économique, c'est la notion de performance qui est mise en avant par la rationalité gestionnaire; et pour l'atteindre, cette dernière exhorte à dissocier la tête de la main dans un mouvement privilégiant l'intelligence abstraite au détriment de l'intelligence concrète - un phénomène illustré par l'évolution du système de missile antichar, mais aussi par la privatisation d'une partie des activités de la DGA.

Ces rationalités conduisent cependant à une série de paradoxes. Sans tendre à l'exhaustivité, citons-en quelques-uns. Tout d'abord, le rapport qu'entretient actuellement notre société à la mort et à la guerre, pourrait-on ajouter - pousse à instaurer des règlements ou à penser les matériels de façon à ce que la sécurité du soldat prime, au point que les combattants se sentent parfois gênés dans la réalisation de leur mission.
Ensuite, les méthodes employées en conception reposent sur le postulat de la simplicité, mais les usagers et les concepteurs qualifient le résultat de "complexe". Il l'est parce que la complexité se niche dans la négation de la dimension qualitative du réel, négation qui est au cœur du postulat de la simplicité. Le pendant de cette complexité technique est la complexité relationnelle, dont témoignent la place croissante des ergonomes ou encore le besoin d'instaurer un langage commun entre DGA et industriels.

En outre, bien que la finalité des approches mises en œuvre soit de mieux faire face à l'incertain, les ingénieurs du public et du privé admettent avoir perdu en capacité d'appréhension du fonctionnement de l'ensemble, du moins par leurs seules capacités intellectuelles - la modélisation et la simulation viennent compenser cette limite. Et de même que l'objectif des concepteurs est d'être prêt à toute éventualité, les combattants souffrent d'une moins grande adaptabilité - et c'est là notre quatrième paradoxe. En effet, la polyvalence d'un matériel n'est pas étroitement liée à son degré de technicité, mais repose sur des caractéristiques sociales (qui recoupent largement les conditions de l'appropriation) et sur des caractéristiques techniques. Or, les choix qui sont faits en amont ferment les objets techniques au sens de Simondon ${ }^{10}$ et sapent l'autonomie du collectif.

Enfin, la puissance manifestée par la rationalité gestionnaire et technicienne apparaît à la fois réelle et simulée, bien là et en même temps fragile. On peut y voir deux explications : la distanciation des réalités militaires et de la culture militaire qui affecte les concepteurs participe d'une élaboration d'armements hors sol (hors temps, hors espace), et d'une déréalisation de l'ennemi. Par ailleurs, la vision fonctionnalisante du travail des combattants néglige la part jouée par l'intelligence concrète, que les militaires appellent "intelligence de situation » mais qu'on peut aussi assimiler à la ruse, aux arts de faire, ou encore à la mètis ${ }^{11}$. 
À cet égard, la faible implication des usagers dans le processus décisionnel autorise les concepteurs à leur attribuer un rôle d'exécutants, pièces du système technique qu'ils ont bâti et qui doit procurer un maximum de performance technique. À ce titre, le fossé entre concepteurs et usagers constitue un point d'achoppement. On peut relever trois freins à leur rapprochement : d'abord le but lucratif des entreprises de défense considéré comme difficilement compatible avec le sens du service public et de l'intérêt général, ensuite l'importance attribuée à la rupture technologique, que les ingénieurs sont a priori mieux à même d'atteindre que les combattants, et enfin l'imaginaire du contrôle, hostile aux développements individuels et locaux.

Sur la base de ces résultats, quelques axes pourraient être approfondis. Mes recherches ont montré que le choix du high-tech ne relevait pas tant de considérations opérationnelles, contrairement à ce qui est avancé spontanément par les acteurs et en particulier les concepteurs, mais que les choix techniques étaient fortement influencés par tout un imaginaire entourant le progrès et la technique. L'étude de cas du système antichar Milan ayant montré qu'il n'y avait pas de nécessaire dépendance entre le niveau technique d'un matériel d'un côté, et la polyvalence et le sentiment de performance de l'autre, cela ouvre des perspectives pour penser différemment la performance des matériels, et invite à associer les usagers à cette réflexion - ce qui prolonge d'une certaine façon le débat sur les relations entre militaires usagers et scientifiques concepteurs initié dans l'entre-deux guerres.

En parallèle, je souhaiterais poursuivre ma réflexion sur les caractéristiques techniques favorisant une réinvention des matériels, afin de parvenir à mieux conceptualiser les deux sortes de polyvalence qui me sont apparues : la polyvalence fonctionnelle et la polyvalence sociale. Enfin, puisque certains choix peuvent affaiblir la puissance manifestée, il me semble indispensable d'inclure, dans une réflexion sur l'équipement, une réflexion sur ce qui procure une puissance solide.

Par ailleurs, pour sortir du strict cadre francofrançais, il était initialement prévu d'inclure une comparaison entre la France et le Royaume-Uni comparaison abandonnée faute de temps. Celle-ci me paraît pertinente dans la mesure où les deux pays partagent les mêmes ambitions politiques internationales, sont les deux plus importants contributeurs européens en matière de recherche et développement militaire, et font face à la même difficulté budgétaire. De plus, l'absence de coupure franche entre monde opérationnel et monde de l'ingénierie au Royaume-Uni aurait permis d'estimer les effets d'un rapprochement entre concepteurs et usagers.

Pour conclure, je tiens à préciser que ce travail ne s'oppose pas aux avancées techniques, mais constitue une réflexion sur ce qu'on peut attendre de la science et de la technique. Cette interrogation m'apparait d'autant plus essentielle que le modèle d'équipement actuel n'est pas pérenne : des savoirfaire se dégradent ${ }^{12}$, les taux de disponibilité des matériels en métropole fluctuent sauf exceptions entre $40 \%$ et $60 \%{ }^{13}$, et les coûts de maintenance restent élevés en dépit des restructurations. Cet état des lieux nécessite que des choix clairs et constants soient faits pour conduire une remontée en puissance, et cela au niveau politique, économique mais aussi technique. À cet égard, ma thèse se veut une contribution pour relever ce défi.

Monsieur le président, Mesdames et Messieurs les membres du jury, je vous remercie de votre attention.

\footnotetext{
${ }^{1}$ La notion de technicité est ici entendue au sens habituel comme « un degré de complexité ou de simplicité sous le rapport des équipements et des connaissances nécessaires à leur conception et à leur maîtrise » (Collectif, André Leroi-Gourhan ou les Voies de l'homme: Actes du colloque du C.N.R.S en mars 1987, Albin Michel, 1988).
} 
${ }^{2}$ David Edgerton remarque que les études sur l'entretien sont " marginales » dans Quoi de neuf? Du rôle des techniques dans l'histoire globale, Paris : Seuil, 2013, p.117.

3 Mc KENZIE Donald, Inventing accuracy : a historical sociology of nuclear missile guidance, Cambridge: The MIT Press, 1990.

4 GENYIES William (dir.), Le choix des armes : théories, acteurs et politiques, Paris : CNRS Éditions, 2004.

5 FLICHY Patrice, " La place de l'imaginaire dans l'action technique », Réseaux - techniques et imaginaires, $\mathrm{n}^{\circ} 109$,

Hermès Science Publication, 2001, p.71.

${ }^{6}$ WEBER Max, Le Savant et le politique, Paris : Plon, 1959.

7 MAUSS Marcel, Manuel d'ethnographie, Paris : Payot, 1947.

${ }^{8}$ MORIN Edgarn, Introduction à la complexité, Paris : Seuil, 2005 .

9 ARENDT Hannah, La Condition de l'homme moderne, Paris : Agora Pocket, 2008.

10 SIMONDON Gilbert, $\mathrm{Du}$ mode d'existence des objets techniques, Paris : Aubier-Montaigne, 1969, pp. $247 \mathrm{~s}$.

${ }^{11}$ DETIENNE Marcel et VERNANT Jean-Pierre, Les Ruses de lintelligence, la Mètis des grecs, Paris: Flammarion, coll. « Champs », 1974.

${ }^{12}$ Le député M. Jean-Claude Viollet affirme que «Par manque de disponibilité des matériels, lié à leur vétusté, le largage à très faible hauteur ne [peut] plus être assuré comme nous le souhaiterions et comme nos engagements le nécessiteraient », et «faute de pouvoir s'entraîner, les compétences reculent dans le domaine tactique ». Évoquant un document que lui aurait remis l'armée de l'air portant sur "la perte des compétences dans le temps, à moyens constants ", il explique qu' « [à] ce jour [28 octobre 2009], l'armée de l'air estime d'ores et déjà avoir perdu la compétence de largage à basse altitude et le processus est engagé pour la direction de dispositifs aériens complexes, du type évacuation de ressortissants ou ponts humanitaire. Ce sont là des aptitudes lentes à acquérir et qui peuvent être très rapidement perdues». "nous n'avons qu'un seul C160 disponible 5 semaines par an pour procéder à des largages à très faible hauteur", explique le député dans le CR $\mathrm{n}^{\circ} 12$, 28/10/2009, p.19.

${ }^{13}$ Olivier Berger, «L'âge des équipements français et le coût du maintien en condition opérationnelle », http://defense.blogs.lavoixdunord.fr/archive/2014/08/06/mco- depiute-13240.html , Défense Globale, blog de «La Voix du Nord », 6 août 2014 . 